\title{
Parallel Estimation and Control Architectures for Deep-Space Formation Flying Spacecraft
}

\section{Conference Paper}

Author(s):

Smith, Roy (D); Hadaegh, Fred Y.

Publication date:

2006

Permanent link:

https://doi.org/10.3929/ethz-b-000476974

Rights / license:

In Copyright - Non-Commercial Use Permitted

Originally published in:

https://doi.org/10.1109/AERO.2006.1655996 


\title{
Parallel Estimation and Control Architectures for Deep-Space Formation Flying Spacecraft
}

\author{
Published in: Proc. IEEE Aerospace Conf., Big Sky, MT, USA, 2006. \\ doi: 10.1109/AERO.2006.1655996. C2006 IEEE \\ Roy S. Smith \\ Dept. of Electrical \& Computer Engineering, \\ University of California, \\ Santa Barbara, CA 93106, USA.$$
\text { +1 (805) 893-2967 }
$$$$
\text { royeece.ucsb.edu }
$$ \\ and \\ Fred Y. Hadaegh \\ Jet Propulsion Laboratory, \\ California Institute of Technology, \\ 4800 Oak Grove Dr., \\ Pasadena, CA 91109, USA. \\ +1 (818) 354-8777 \\ Fred.Y.Hadaeghejpl.nasa.gov
}

\begin{abstract}
The formation flying of precisely controlled spacecraft in deep space can be used to implement optical instruments capable of imaging planets in other solar systems. The distance of the formation from Earth necessitates a significant level of autonomy and each spacecraft must base its actions on its estimates of the location and velocity of the other spacecraft. Precise coordination and control is the key requirement in such missions and the flow of information between spacecraft must be carefully designed. Doing this in an efficient and optimal manner requires novel techniques for the design of the on-board estimators. The use of standard Kalman filterbased designs can lead to unanticipated dynamics-which we refer to as disagreement dynamics-in the estimators' errors. We show how communication amongst the spacecraft can be designed in order to control all of the dynamics within the formation. We present several results relating the topology of the communication network to the resulting closed-loop control dynamics of the formation. The consequences for the design of the control, communication and coordination are discussed.
\end{abstract}

\section{INTRODUCTION}

Precision spacecraft formations provide the ability to synthesize science instruments of greater capability than can be achieved with a single spacecraft. In applications where the size of synthesized instrument correlates with performance, spacecraft formations are critical to the mission. Interferometric imaging is a prototypical example of such an application, and is the primary motivation for the work described here. Spaceborne interferometers have by proposed over the last two decades [1], [2], [3], [4], [5], and several space flight mission project-based on formation flying - have been proposed. Examples include Darwin [6], LISA [7], Starlight (formerly ST-3) [8], Terrestrial Planet Finder (TPF) [9], Stellar Imager [10], Microarcsecond X-ray Imaging Mission (MAXIM) [11], Submillimeter Probe of the Evolution of Cosmic Structure (SPECS) [12], and X-ray Evolving Universe Spectroscopy (XEUS) [13].

The interferometric imaging application is illustrated conceptually in Figure 1. Each spacecraft is equipped with the equivalent of an optical "bench" and, acting as a light collector, reflects light from the deep space imaging target to a combiner spacecraft. The combiner spacecraft can generate an interference pattern between the beams from collectors within the formation. It also contains optical delay lines enabling the optical path lengths between the collectors to be manipulated. Measuring the amplitude and phase (with respect to equal optical path lengths) of the peak of the pattern is equivalent to taking a sample of the spatial Fourier transform of the deep space image. Manoeuvring the formation-or using different collector beam combinations-and taking multiple such samples gives sufficient information to reconstruct to target image. Multiple collectors also allow the creation of nulls in the image response and this can be used to effectively mask bright light sources that would otherwise overwhelm the image. The most obvious application of these techniques is the masking of the light from a distant star to allow the imaging of a planet around that star [14]. As with any optical imaging system the resolution will depend on the effective aperture. The resolutions required for imaging distant solar systems require effective apertures of the order of kilometers. Formation flying spacecraft are clearly required for such systems. See [15] for illustrative examples of other interferometric imaging systems.

The most critical control aspect is the maintenance of the optical path-length while acquiring and measuring the interference pattern. This depends on the relative position of the spacecraft in the formation; their absolute position in space is of far less importance. This is particularly true when considering deep space missions and extra-solar interferometric imaging systems would be designed for heliocentric orbit. To maintain the imaging performance of the formation in deep space missions it is necessary only to maintain the relative positions and absolute orientations of the spacecraft. However, the requirements on relative path length are very stringent; it must be held constant to the order of a small fraction of the wavelength of the light. This is approximately ten nanometers for optical imaging systems.

The deep space aspects simplify the problem somewhat- 


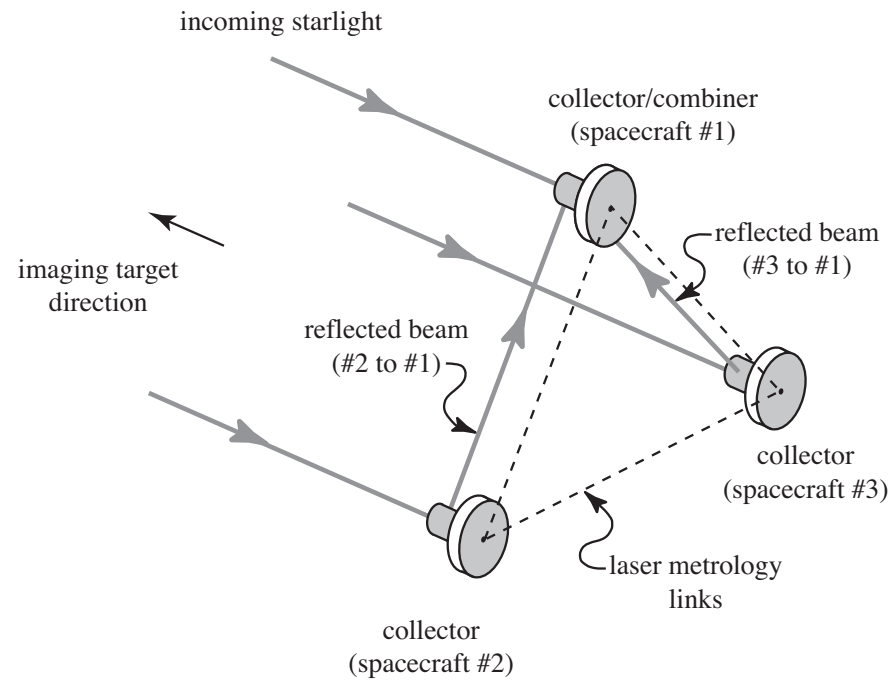

Fig. 1. An formation flying based interferometric imaging configuration. The spacecraft may be separated by distances which range between tens of meters to kilometers.

one need not model in detail the orbital effects that would dominate the problem in an earth orbiting scenario-but also leads to additional complications: the absolute position measurements are either unavailable or of very low accuracy. Another consideration of the deep space application is that the dynamics of the spacecraft are coupled only through their common objective of maintaining or manoeuvring the formation. Highly accurate relative position measurements are available and in developing a mathematical model, this also couples the dynamics of the spacecraft.

Our emphasis in this paper is on the formation control topology at the level of precision coordination between the spacecraft, rather than the detailed actuation and sensing systems within a spacecraft. Mission and trajectory planning functions are carried out at the systems level above that considered here. The focus on deep space missions allows us to address formation flying problems at the key level of inter-spacecraft communication and coordination.

Hierarchical actuation is used to achieve the stringent optical path-length requirements. Various actuators may been used and these include: movable platforms, optical delay lines, adaptive optics, precision piezoelectric actuation and deformable mirrors. The work presented here considers the actuation in a generic form, at the level of the spacecraft positions. This typically gives relative position requirements in the micrometer to centimeter range [6], [9]. The specific details of the actuation is highly application dependent and beyond the scope of the work presented here. We will consider each spacecraft to be equipped with force actuators in three dimensions and torque actuators capable of control of the orientation.

Similar considerations apply to the formation sensing. Several technologies are available for the measurement of the relative positions of the spacecraft. For example, the Autonomous Formation Flying Sensor (AFF) [16] uses a GPS based architecture with several transmitters and receivers mounted on each spacecraft, to give relative position measurements to $\pm 10 \mathrm{~mm}$, relative velocity measurements to $\pm 0.1 \mathrm{~mm} / \mathrm{sec}$. Laser metrology can be used to give relative measurements accurate to $\pm 10 \mathrm{~nm}$ or better. For the purposes of this paper we consider only a generic relative position (and possibly velocity) measurement in three dimensional distance coordinates.

A wide variety of control topologies have been considered for formation flying problems, both in the Earth-orbiting and deep space mission scenarios. Leader-follower topologies have been studied by numerous researchers. See for example [17], [18], [19], [20], [21], [22], [23], [24]. Such approaches are perhaps the easiest to implement but care must be taken with regard to string-stability if there are more than a few vehicles in the leader-follower chain [25], [26]. Graph theory approaches enable more general communication topologies to be studied [27], [28], [29], and some elements of graph theory will be used here to develop a framework for tradeoff studies between communication topologies. Distributed estimation topologies are a key aspect of the work described here and previous work in this area can be found in [30], [31]. The literature on coordination and control of spacecraft formations is more extensive than the above referencing. See [32] for a more detailed survey.

In the following we present a case for the use of decentralized topologies for the implementation of the coordination and control of the formation. In such topologies the information is distributed amongst the spacecraft in an overlapping or parallel manner. As well as providing structural redundancy, this approach also gives each spacecraft sufficient information to enable it to coordinate complex manoeuvres with other spacecraft. Decentralized control of large scale systems has been considered within the academic control community for some time. See [33], [34], [35] and the references therein for a further detail on decentralized control and estimation, particularly with regard to spacecraft formations.

The approach presented here should be contrasted with a centralized topology in which all measurements are sent to a specified spacecraft, and that spacecraft calculates the required control action for all members of the formation. The control actions are then communicated back to the individual members before being carried out. This approach clearly introduces a latency into the feedback loop leading to performance limitations. It also suffers from single point of failure reliability problems. Communication bandwidth and synchronization constraints lead us to favor topologies which limit the amount of time critical information that must be communicated between spacecraft in the formation. Our work gives a basis for studying these control performance and communication trade-offs.

Two approaches to the formation flying control problem are described in this paper. Underlying both is the view that the control of the formation should be specified in terms of a formation-wide control objective. This follows naturally from the fact that it is the relative positions of the spacecraft which are critical to the performance of the entire formation. The resulting control algorithm is therefore specified in terms of the complete formation state or the complete set of relative 
measurements. The key issue then becomes implementing this algorithm in a distributed manner.

The first approach, described in Section III, involves exploiting the redundancy in the set of relative position measurements. This allows us to develop a family of equivalent decentralized controllers, each using a subset of the relative position measurements. The individual spacecraft may switch asynchronously between these controllers exploiting the readily available relative position measurements. Each spacecraft carries sufficient information to predict line-of-sight measurement occlusions occurring during a manoeuvre and may be able to use communicated measurement information in place of locally available measurements. Decentralized control implementations in aerial formations has been studied in a similar manner in [36], although their approach imposes structural constraints at the design stage.

The second approach, described in Section IV, employs parallel estimators on each of the spacecraft. Each spacecraft maintains an estimate of the behavior of all of the others within the formation and uses this estimate to calculate its component of the formation-wide control algorithm. Doing this requires some care as the formation can exhibit hidden dynamics arising from the disagreement between each spacecraft's estimate of the formation state. Communication amongst the spacecraft can be used to ameliorate this effect and we discuss this in detail in Section V. The tools described here allow us to study the trade-offs between communication topology and formation control performance.

\section{Formation Flying in DeEp Space}

\section{A. Architectural considerations}

There are several aspects of the deep space formation flying problem drive the technological approaches. Many of these also apply in other application domains. These are as follows:

1) Precision and limited fuel. Both of these aspects require that optimality is important in our control architecture. This leads us to pose the control problem in terms of formation-wide objectives. Control and implementation methodologies which address the optimality of resulting formation performance are essential.

2) Relative sensing. The relative positions and velocities of the spacecraft in the formation can be measured with high accuracy. Absolute position measurements are of very low quality if available at all. Fortunately most formation objectives can be effectively posed in terms of relative variables within the formation.

3) A high level of formation autonomy. The significant distance from Earth necessitates control and supervisory architectures that can function with minimum intervention by ground-based operators. The execution of supervisory level formation manoeuvres should be managed and controlled by the formation itself.

4) Multiple roles in a reliable architecture. The functioning of the formation should not be critically dependent on an individual spacecraft. Although this is more determined by hardware considerations, the control architecture should allow role switching in the event of a failure. Broadly speaking, information should be distributed amongst the spacecraft in the formation, and should also be redundant.

5) Computation versus communication. In trading off between additional computational resources and additional communication channels between spacecraft; computation is significantly cheaper. However, being able to quantify the potential performance gain from the addition of communication channels is essential.

These considerations favor a distributed estimation and control architecture as opposed to a more strictly hierarchical structure. We propose a parallel estimation structure which provides each spacecraft access to an estimate of the complete formation state. This, together with a knowledge of the control algorithms on the other spacecraft, allows individual spacecraft to implement their component of formation-wide algorithms. This may also involve higher level functions such as initialization, formation reconfiguration or retargeting, and collision avoidance.

\section{B. Notation}

Before proceeding with the mathematical formulation of the control problem, we will outline the notation used to simplify some of the statements.

The $N \times N$ identity matrix is denoted by $I_{N}$, and $1_{N}$ will be used to indicate a column vector of ones of dimension $N$. For notational convenience we will make frequent use of the Kronecker product,

$$
X \otimes Y:=\left[\begin{array}{cccc}
X_{1,1} Y & X_{1,2} Y & \ldots & X_{1, n} Y \\
X_{2,1} Y & X_{2,2} Y & & \\
\vdots & & \ddots & \vdots \\
X_{m, 1} Y & X_{m, 2} Y & \cdots & X_{m, n} Y
\end{array}\right] .
$$

\section{Problem description}

To describe the architecture and algorithms we introduce the following variables and notation. Figure 2 illustrates the definition of the position and orientation variables. There are $N$ spacecraft and the position of the $\imath$ th spacecraft, with respect to a local inertial frame, is the three dimensional vector, $p_{i}$. Each spacecraft has orientation $\phi_{i}$ with respect to a reference direction, $\phi_{\text {ref }}$.

In deep space applications the formation objectives may be specified in terms of the relative positions,

$$
r:=\left[\begin{array}{llllllll}
r_{12} & \cdots & r_{1 N} & r_{23} & \cdots & r_{i j} & \cdots & r_{N-1 N}
\end{array}\right]^{T},
$$

where,

$$
r_{i j}=p_{j}-p_{i}, \quad i=1, \ldots, N-1, \quad j=2, \ldots, N, \quad i<j,
$$

and individual spacecraft orientations, $\phi_{i}, i=1, \ldots, N$. When discussing the time-dependent aspects of these variables we will include a discrete-time index: $r(k)$, for example. We note that each spacecraft is typically instrumented with local sensing and actuation for orientation. The control of orientation therefore naturally decomposes into local controllers. This is not true of the control of relative position as the movement of 


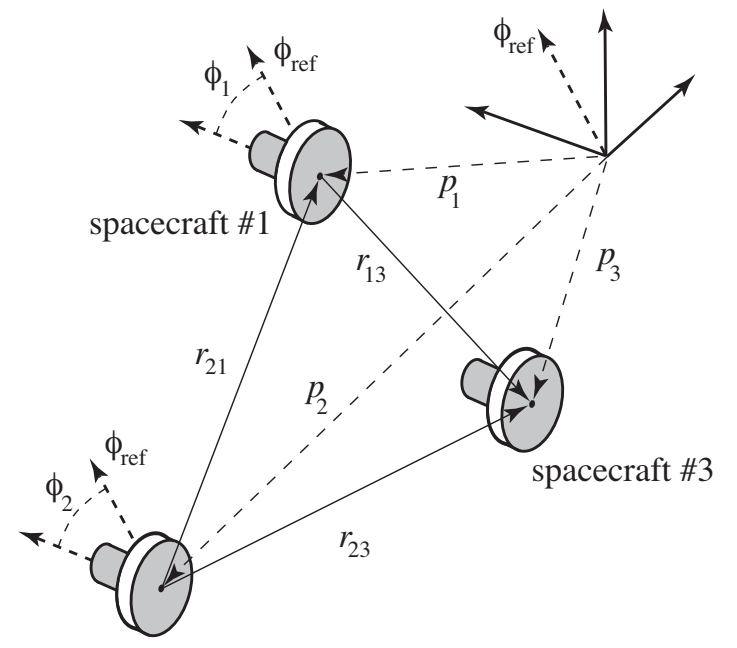

spacecraft \#2

Fig. 2. Formation position and orientation variables. Both absolute and relative position variables are shown. The position of the local inertial reference is far less important than the relative positions of the spacecraft.

a single spacecraft will alter $N-1$ of the $N(N-1) / 2$ relative position variables. ${ }^{1}$ We therefore focus on the more difficult problem of relative position control.

The dynamics of the spacecraft are coupled only by the measurement and performance objectives. By collecting the state-space representations, the complete formation dynamics are described-in an inertia coordinate frame-by,

$$
\begin{gathered}
x(k+1)=\left[\begin{array}{ccc}
A_{1} & & 0 \\
& \ddots & \\
0 & & A_{N}
\end{array}\right] x(k)+\left[\begin{array}{ccc}
B_{1} & & 0 \\
& \ddots & \\
0 & & B_{N}
\end{array}\right]\left[\begin{array}{c}
u_{1}(k) \\
\vdots \\
u_{N}(k)
\end{array}\right], \\
p(k):=\left[\begin{array}{c}
p_{1}(k) \\
\vdots \\
p_{N}(k)
\end{array}\right]=\left[\begin{array}{ccc}
C_{1} & & 0 \\
& \ddots & \\
0 & & C_{N}
\end{array}\right] x(k)
\end{gathered}
$$

where $u_{i}$ is the three dimensional actuation command applied by the $\imath$ th spacecraft.

The decoupled structure in (1) and (2) does not remain when we consider the formation problem in terms of the relative position variables. Expressing the system with the relative positions, $r(k)$, as the output destroys the block diagonal structure of the $C$ matrix. More importantly, a representation in terms of relative positions is not observable; it is not possible to determine the position or velocity of the formation centroid. The solution is to remove the unobservable subspace from the representation. As the formation objectives are specified in terms of the relative positions, removing the unobservable subspace does not affect the achievable control performance. However it does destroy the decoupled structure in both the $A$ and $B$ matrices. See [37] for details on removing unobservable formation dynamics.

For these reasons, our subsequent development will use only a generic state-space model, with the vector of relative

\footnotetext{
${ }^{1}$ We neglect the opposite sign equalities, $r_{i j}=-r_{j i}$, in this count.
}

positions as the output,

$$
\begin{aligned}
x(k+1) & =A x(k)+B u(k)+G w(k), \\
r(k) & =C x(k)+v(k),
\end{aligned}
$$

where $w(k)$ models unmeasured disturbances to the formation, and $v(k)$ models the sensor noise. We will assume that the covariances of the noise signals are $Q_{w}$ and $Q_{v}$ respectively. It should be borne in mind that the state vector, $x(k)$, contains complete state information about all $N$ spacecraft in the formation, and that it is observable from the relative measurement vector, $r(k)$.

\section{Formation-wide control architectures}

The development above leads to a wide variety of potential design methodologies which trade-off between an relative position tracking objective, specified in terms of $r(k)$ and $w(k)$ (or $Q_{w}$ ) and the actuation effort, specified in terms of a cost or constraint on $u(k)$. Many standard methodologies may be used; the problem is easily translated into a Linear Quadratic Gaussian framework for example. The exact nature of the control design is not the thrust of this paper; we are more interested in the dynamical consequences of the architectural choices and for this purpose the key point is that the control design cost is specified in terms of $r(k), x(k), u(k), w(k)$ and $v(k)$.

\section{RELATIVE SENSING BASED CONTROL}

We now consider exploiting the redundancy in the relative position vector to develop a family of stabilizing controllers that will operate in concert to achieve the specified control performance. This approach requires that each spacecraft has access (either local or communicated) to $N-1$ independent relative position measurements. ${ }^{2}$

We assume that a controller has been designed to achieve the formation state objectives outlined above. In the general LTI case this controller has $N(N-1)$ input measurements and $N$ actuation outputs, and can be written as a dynamic controller, $K_{\mathrm{d} y n}$, in the form,

$$
u(k)=\left[\begin{array}{c}
u_{1}(k) \\
\vdots \\
u_{N}(k)
\end{array}\right]=K_{\mathrm{d} y n}\left[\begin{array}{c}
r_{12}(k) \\
\vdots \\
r_{N-1 N}(k)
\end{array}\right]=K_{\mathrm{d} y n} r(k) .
$$

For notational simplicity we define a matrix, $\Pi_{i}$, which simply selects the actuation signals applicable to the $\imath$ th spacecraft. In otherwords,

$$
u_{i}\left(k 0=\Pi_{i}\left[\begin{array}{c}
u_{1}(k) \\
\vdots \\
u_{N}(k)
\end{array}\right]=\Pi_{i} K_{\mathrm{d} y n}\left[\begin{array}{c}
r_{12}(k) \\
\vdots \\
r_{N-1 N}(k)
\end{array}\right] .\right.
$$

This amounts to selecting one or more rows from the matrix valued controller, $K_{\mathrm{d} y n}$. Although, it appears that each spacecraft requires the complete vector of relative position measurements, the redundancy in $r$ can be exploited to use

\footnotetext{
${ }^{2}$ The dimensions specified here apply to a one-dimensional problem. For a three dimensional problem each dimension, or measurement count, is simply multiplied by three.
} 
as few as $N-1$ independent measurements from $r$. The details of this are given in [37] and are summarized below for completeness.

The redundancy in $r$ can be expressed as algebraic constraints, amounting to the fact that all closed paths in $r$ sum to zero. For example,

$$
r_{i j}+r_{j k}+r_{k i}=0, \text { for all } i, j, k .
$$

This can be expressed by defining a right invertible matrix, $M \in \mathcal{R}^{N(N-1) / 2 \times(N-1)(N-2) / 2}$, satisfying, $M^{T} r=0$. The choice of $M$ is not unique.

Suppose that we now select a matrix $X \in$ $\mathcal{R}^{N(N-1) / 2 \times(N-1)(N-2) / 2}$ satisfying, $M^{T} X=I$. Then the transformation,

$$
H=I-X M^{T}
$$

has the effect of replacing one measurement by a linear combination of the others. The choice of measurement, $r_{i j}$, to be replaced is determined by the choice of $X$.

For example, if $H_{j k}$ is constructed to remove $r_{j k}$ from $r$, then this give a result of the form,

$$
H_{j k}\left[\begin{array}{c}
r_{i j} \\
r_{i k} \\
r_{j k}
\end{array}\right]=\left[\begin{array}{c}
r_{i j} \\
r_{i k} \\
r_{i k}-r_{i j}
\end{array}\right]=:\left[\begin{array}{c}
r_{i j} \\
r_{i k} \\
\hat{r}_{j k}
\end{array}\right],
$$

where $\hat{r}_{j k}$ denotes the estimated value of $r_{j k}$. Note that this estimation is algebraic, rather than the more usual dynamic estimation case, and this gives additional properties with respect to stability and tracking performance.

Each controller now uses the appropriate $H$ matrix to reconstruct the unavailable measurements and replace them with algebraic estimates. Controllers may switch asynchronously between subsets of $r$ without compromising the overall formation stability and tracking performance. This is presented in the following theorem (proven in [37]).

Theorem 1: Given a controller, $K_{\mathrm{d} y n}$, with measurement input $r$ and actuation output $u$, that stabilizes the collective formation dynamics in (3) and (4), then, if $K_{\mathrm{d} y n}$ is stable, the asynchronously switched controller,

$$
\hat{K}_{\mathrm{d} y n}=\sum_{i=1}^{N} \Pi_{i}^{T} \Pi_{i} K_{\mathrm{d} y n} H_{i},
$$

where the $H_{i}$ are any switching transformation matrices of the form given in (5), also internally stabilizes the system (3) and (4).

Furthermore the closed-loop transfer function between the reference command and the relative positions is identical for $K_{\mathrm{d} y n}$ and $\hat{K}_{\mathrm{d} y n}$.

As long as each controller has access to $N-1$ independent measurements the complete $r$ vector can be reconstructed in this manner. Note that $N-1$ measurements are defined with respect to each spacecraft and in small formations it may be feasible to locally measure the $N-1$ required independent measurements.

The statement of this theorem requires that $K_{\mathrm{d} y n}$ is itself open-loop stable. This is an unusual constraint and precludes the use of integral control. The reason for this will become

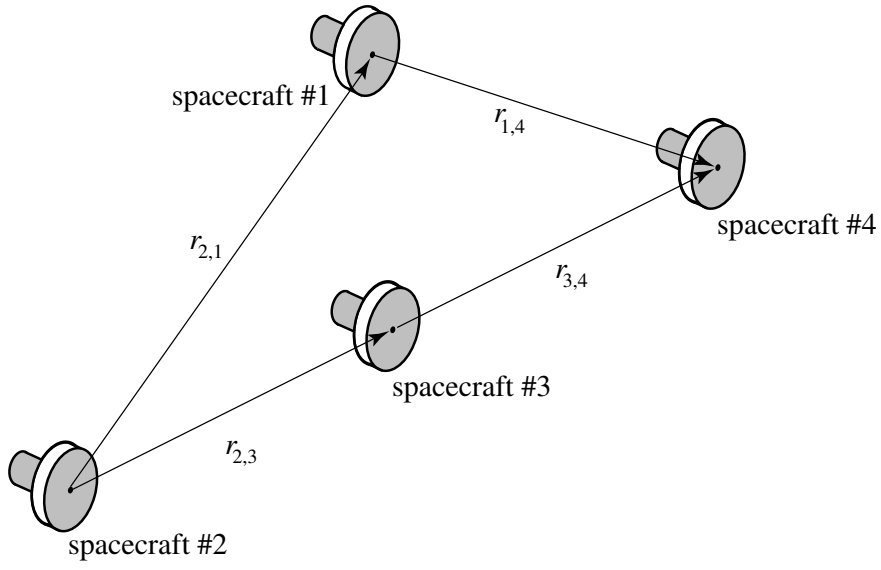

Fig. 3. Line-of-sight measurement occlusion by formation shadowing. Spacecraft \#2 cannot determine $r_{2,4}$ from the line-of-sight measurements: $r_{2,1}$ and $r_{2,3}$. Either $r_{1,4}$ or $r_{3,4}$ must be communicated to spacecraft \#2 to allow reconstruction of $r$. An analogous situation exists for spacecraft \#4.

clear in Section IV. We should also note that the noise response of the system using reconstructed measurements may differ from that using physical measurements.

In certain formation configurations, or during formation manoeuvres, line-of-sight occlusions may block some of the relative measurements. Figure 3 illustrates such an example. In these cases an additional relative position measurement must be communicated from an adjacent spacecraft. Control algorithms are implemented by digital controllers and in order to implement the desired control objective, the communicated information must be received before the next control calculation sample time. This clearly imposes requirements on the communication bandwidth, and may limit this approach to small formations. If communicated measurements must be transferred via multiple hops then bandwidth limitations may constrain the speed at which remote measurement information is available, thereby constraining the formation control performance.

The approach taken in the next section overcomes this limitation by maintaining full formation estimates on all spacecraft. In doing this we are effectively circumventing some of the bandwidth limitations at the expense of requiring greater computational facilities on each spacecraft. As pointed out in the earlier discussion, computation is a less stringent constraint than communication bandwidth. In this sense, the following parallel estimation approach is advantageous in larger formations.

\section{PARAllel ESTIMATION}

Parallel estimation involves implementing a full formation state estimator on each spacecraft and basing each spacecraft's control on its state estimate. This is a more general architecture and can be integrated with communication amongst the spacecraft.

We begin by assuming that a formation-wide optimal control objective has been used to design a state-feedback controller of the form,

$$
u(k)=-K x(k),
$$


yielding a suitable closed-loop state transition matrix,

$$
A_{\text {clp }}:=A-B K \text {. }
$$

Note that $x(k)$ refers to the full formation state, given in (3), and typically includes a transformed version of the relative positions and velocities.

Implementing the feedback control in (6) requires knowledge of the entire formation state. This could be implemented by having all spacecraft transmit their measurement information to a predesignated central spacecraft. The central spacecraft could then calculate $u(k)$ and transmit this back to each of the other spacecraft. Doing this has the disadvantage of introducing an additional round-trip communication time latency into the implementation of the control, thereby limiting the achievable control performance. It also concentrates the information on a single spacecraft, leading to a single point of failure for the formation. The third disadvantage is that the communication requirements, in terms of the number of communication links, grows with the square of the number of spacecraft.

Our preferred approach is to implement a full state estimator on each spacecraft. This is computationally more expensive, but increases the structural reliability of the formation. Spacecraft can more easily reconfigure to switch roles in the event of the loss of a single spacecraft from the formation. It also has the advantage of avoiding the round-trip communication time latency in the control. Effective implementation of this method still requires some communication, however we will demonstrate that the number of communication links need only grow linearly with the number of spacecraft in the formation.

Having an estimator on-board each spacecraft also provides another significant benefit. Each spacecraft "knows" the relative positions of all of the others and can implement preprogrammed algorithms for the execution of higher level formation functions such as reconfiguration, retargeting, and collision avoidance. This increases the overall autonomy of the formation, reducing the level of interaction required with earth-based operators.

Define $r_{i}(k)$ as those components of $r(k)$ that the $\imath$ th spacecraft can measure directly. In general these will be corrupted by noise $v_{i}(k)$ and can be expressed in terms of the full formation state as,

$$
r_{i}(k)=C_{i} x(k)+v_{i}(k) .
$$

We can express the estimator on the $\imath$ th spacecraft in the form,

$$
\begin{aligned}
\hat{x}_{i}(k+1) & =\left(A-B K-L_{i} C_{i}\right) \hat{x}_{i}(k)+L_{i} r_{i}(k) \\
u_{i}(k) & =\Pi_{i} K \hat{x}_{i}(k) .
\end{aligned}
$$

The notation $\hat{x}_{i}$ is used to denote the estimate residing on the $\imath$ th spacecraft. The error in this estimate is defined as,

$$
e_{i}(k):=x(k)-\hat{x}_{i}(k) .
$$

In the single estimator case the error dynamics are given by the error state-transition matrix, $A-L C$. In the parallel estimator case the situation is more complex. A part of the problem comes from the fact that the $\imath$ th spacecraft does not know the actuation commands of the other spacecraft. The best that it can do is estimate those actions on the basis of its own estimate of the state, and a preprogrammed knowledge of the other spacecrafts' control algorithms. As the following section will show, the use of estimated actuation signals leads to disagreement dynamics.

\section{A. Disagreement dynamics}

We now consider the dynamics of the complete system: $N$ implementations of the estimators given in (7) and (8), plus the physical states of the formation given in (3) and (4). For simplicity we will assume that each spacecraft has access to the same subset of measurements of $r(k)$ and that the formation state, $x(k)$, is observable from that subset. ${ }^{3}$

If we were to design each estimator as a Kalman filter, the single estimator error dynamics, $A-L C=: A_{\text {est }}$, would be optimal for the given covariances, $Q_{w}$ and $Q_{v}$. However the complete system dynamics would be of the form:

$$
\begin{aligned}
{\left[\begin{array}{c}
x(k+1) \\
e_{1}(k+1) \\
\vdots \\
e_{N}(k+1)
\end{array}\right]=} & \\
& \left(\left[\begin{array}{cc}
A_{\text {clp }} & 0 \\
0 & I_{N} \otimes A_{\text {ctrl }}
\end{array}\right]+R\right)\left[\begin{array}{c}
x(k) \\
e_{1}(k) \\
\vdots \\
e_{N}(k)
\end{array}\right] .
\end{aligned}
$$

where, $A_{\text {ctrl }}:=A-B K-L C$, and

$$
R=\left[\begin{array}{cccc}
0 & B \Pi_{1}^{T} \Pi_{1} K & \cdots & B \Pi_{N}^{T} \Pi_{N} K \\
0 & B \Pi_{1}^{T} \Pi_{1} K & \cdots & B \Pi_{N}^{T} \Pi_{N} K \\
\vdots & \vdots & & \vdots \\
0 & B \Pi_{1}^{T} \Pi_{1} K & \cdots & B \Pi_{N}^{T} \Pi_{N} K
\end{array}\right] .
$$

For simplicity we have dropped the effect of the noise terms as they do not affect the closed-loop stability result that we now present. The equilibrium dynamics of this system are not simply the closed-loop plant dynamics $\left(A_{\mathrm{clp}}\right)$ and the estimator error dynamics $\left(A_{\mathrm{est}}\right)$ as would be the case in the single estimator problem. The following theorem (proven in [38]) shows that the system stability also depends on the eigenvalues of $A_{\text {ctrl }}$.

Theorem 2: Given a plant, described by (3) and (4), and $N$ parallel estimators, each defined by (7) and (8), the closed-loop system eigenvalues are:

$$
\operatorname{eig}\left(A_{\text {clp }}\right) \cup \operatorname{eig}\left(A_{\text {est }}\right) \cup \underbrace{\operatorname{eig}\left(A_{\text {ctrl }}\right) \cup \cdots \operatorname{eig}\left(A_{\text {ctrl }}\right)}_{N-1 \text { times }} .
$$

Clearly, closed-loop stability of the system now also depends on the controllers' open-loop dynamics: $A_{\text {ctrl }}$. We refer to these $N-1$ copies of the controllers' dynamics as disagreement dynamics as they actually drive the difference in estimates between each of the spacecraft. Note that the

\footnotetext{
${ }^{3}$ This choice is for simplicity in the discussion. The situation where each spacecraft uses a different subset of $r(k)$ also exhibits disagreement dynamics although they are not as cleanly expressed.
} 
standard design methods do not account for the additional disagreement dynamics, which leads to a loss of performance, and may even give rise to instability in the formation.

One remedy is to communicate each spacecraft's actuation command, $u_{i}(k)$, to all other spacecraft in the formation. As shown in the following theorem (also proven in [38]), this restores the system dynamics to those predicted by the initial design methodology.

Theorem 3: If each estimator/controller communicates its actuation input signal,

$$
u_{i}(k)=\Pi_{i} K \hat{x}_{i}(k), \quad i=1, \ldots, N,
$$

to all other estimator/controllers, then the closed-loop system eigenvalues are:

$$
\operatorname{eig}\left(A_{\text {clp }}\right) \cup \underbrace{\operatorname{eig}\left(A_{\text {est }}\right) \cup \cdots \cup \operatorname{eig}\left(A_{\text {est }}\right)}_{N \text { times }} .
$$

The disadvantage of this approach is that $N(N-1)$ communication links are now required in this topology. The fact that the communication requirements grow with the square of the number of spacecraft precludes using this approach in large formations. Section V will show that there exists a more efficient, linear growth, communication topology having the same result. An alternative is to consider the estimation problem in terms of the full system dynamics given in (9) and this is done in the next section.

\section{B. Structured estimation}

Kalman filter based single estimator designs are well understood but in a formation context, suffer from the disagreement dynamics problem illustrated above. We can address this directly by designing the estimators for the complete structure in (9). The drawback is that the resulting design problem imposes additional structural constraints on the Kalman filter solution. These constraints have not been well studied. In the following we pose the structured design problem. We have developed several approaches for solving this problem-both in a time-invariant and a time-varying context-and these are outlined in [39].

We can express the complete formation state and estimation error update equations in the form,

$$
\begin{gathered}
{\left[\begin{array}{c}
x(k+1) \\
e_{1}(k+1) \\
\vdots \\
e_{N}(k+1)
\end{array}\right]=\left(\left[\begin{array}{cc}
A_{\mathrm{clp}} & 0 \\
0 & I_{N} \otimes A_{\mathrm{clp}}
\end{array}\right]-\left[\begin{array}{cc}
0 & 0 \\
0 & L_{f} C_{f}
\end{array}\right]\right.} \\
\left.+\left[\begin{array}{ll}
0 & M_{1} \\
0 & M_{N}
\end{array}\right]\right)\left[\begin{array}{c}
x(k) \\
e_{1}(k) \\
\vdots \\
e_{N}(k)
\end{array}\right]+\left[\begin{array}{cc}
G & 0 \\
\Gamma & -L_{f}
\end{array}\right]\left[\begin{array}{c}
w(k) \\
v_{1}(k) \\
\vdots \\
v_{N}(k)
\end{array}\right],
\end{gathered}
$$

where,

$$
L_{f}=\left[\begin{array}{ccc}
L_{1} & & 0 \\
& \ddots & \\
0 & & L_{N}
\end{array}\right], \quad C_{f}=\left[\begin{array}{ccc}
C_{1} & & 0 \\
& \ddots & \\
0 & & C_{N}
\end{array}\right],
$$

$$
\Gamma=1_{N} \otimes G, \quad M_{1}=\left[\begin{array}{llll}
B \Pi_{1}^{T} \Pi_{1} K & \cdots & B \Pi_{N}^{T} \Pi_{N} K
\end{array}\right],
$$

and $M_{N}=1_{N} \otimes M_{1}$. At this point the distributed parallel estimation design problem can be formulated. Note that the estimator error dynamics are separated from the plant dynamics, allowing us to express the error update equations as,

$$
\begin{aligned}
e_{i}(k+1)= & \left(A-B K-L_{i} C_{i}\right) e_{i}(k) \\
& +B \sum_{j=1}^{N} \Pi_{j}^{T} \Pi_{j} K e_{j}(k)+G w(k)-L_{i} v_{i}(k) .
\end{aligned}
$$

for $i=1, \ldots, N$. By considering all $N$ equations simultaneously this can be viewed as a Kalman filter design problem with the additional constraint that the Kalman filter be block diagonal.

The imposition of the structural constraint may lead to a performance trade-off in the estimator design procedure. However, implementing an estimator structure without this constraint is equivalent to having each spacecraft communicate its state estimate to all other spacecraft, again leading to a growth in communication requires that scales with the square of the number of spacecraft.

\section{DYNAMICAL AFFECTS OF COMMUNICATION}

We now examine the disagreement dynamics more closely and determine the minimum number of communication links required to remove the disagreement dynamics from the system.

\section{A. Communication framework}

We will now develop a framework for studying the effects of communicated signals on the closed-loop formation dynamics. The communication links are considered to be unidirectional with the communicated signal defined as a linear function of the transmitting spacecraft's full state estimate. We have $M$ such links,

$$
s_{l}(k)=H_{l} \hat{x}_{l}(k)+z_{l}(k), \quad l=1, \ldots, M,
$$

where $s_{l}(k)$ is the received signal on each link and $z_{l}$ models the channel noise on each communication link. The matrix, $H_{l}$, can be viewed as a transmission gain. This is a simplistic model of a communication channel but it serves to determine the fundamental relationship between the communicated state information and estimator error dynamics.

Each spacecraft receiving the noisy signal, $s_{l}$, must update its own full state estimator. Assuming that the $i$ th estimator receives the $l$ th communicated signal, the estimator is updated via,

$$
\begin{aligned}
\hat{x}_{i}(k+1)=A_{\mathrm{ctrl}} \hat{x}_{i}(k)+ & L r_{i}(k) \\
& +F_{l} s_{l}(k)-F_{l} H_{l} \hat{x}_{i}(k) .
\end{aligned}
$$

Note that noise enters the estimator through both the plant measurement, $y_{i}(k)$, and the received signal, $s_{l}(k)$. The matrix $F_{l}$ can be viewed as the receiver gain for the $l$ th communication link. The differential application of the information in $s_{l}(k)$ has the the effect of maintaining the separation 
between the plant's closed-loop poles and the estimators' error dynamics.

If a spacecraft receives multiple signals the update equations will simply be summed over all received signals and have the form,

$$
\begin{aligned}
\hat{x}_{i}(k+1)= & A_{\text {ctrl }} \hat{x}_{i}(k)+L r_{i}(k) \\
& +\sum_{l=1}^{M_{i}} F_{l} H_{l}\left(\hat{x}_{l}(k)-\hat{x}_{i}(k)\right)+\sum_{l=1}^{M_{i}} F_{l} z_{l}(k),
\end{aligned}
$$

Before we can analyze the closed-loop effects of the communicated signals we introduce a graph theoretical notation for specifying the communication links between spacecraft.

\section{B. Graph theoretical communication topology specification}

Graph theoretical approaches are also used to analyze communication topologies for cooperative vehicles and other coupled systems. See for example [40] and [41].

An adjacency matrix, $\mathcal{A}$ specifies the communication links with $\mathcal{A}_{i, j}=1$ if there is a communication link from spacecraft $j$ to spacecraft $i$. In graph theoretical terms, each spacecraft is represented by a node in the graph. The number of incoming communication links for each spacecraft (or node) is called the in-degree, and the Laplacian of the graph is defined as,

$$
\mathcal{L}:=\mathcal{D}-\mathcal{A}
$$

where $\mathcal{D}$ is a diagonal matrix with the in-degree of each node on the diagonal. It is easy to show that every Laplacian has at least one zero eigenvalue, and with this definition, the sum of Laplacians is also a Laplacian.

To specify the $M$ communication links above we associate each one with a Laplacian, $\mathcal{L}_{l}, \quad l=1, \ldots, M$. We can rewrite the state transition matrix for the complete system dynamics in terms of these Laplacians as,

$$
\left(\left[\begin{array}{cc}
A_{\text {clp }} & 0 \\
0 & I_{N} \otimes A_{\text {ctrl }}-\sum_{l=1}^{M} \mathcal{L}_{l} \otimes F_{l} H_{l}
\end{array}\right]+R\right)
$$

where, $R$ is given by (10). Analyzing the equilibrium disagreement dynamics is simply a matter of determining the eigenvalues of (12).

Several cases are illustrative and potentially useful in a spacecraft formation communication topology. First, we consider the case where the links have identical transmitter and receiver gains, $H$ and $F$ respectively. In this case a single Laplacian, $\mathcal{L}$, can be used to describe all of the links. We denote the eigenvalues of $\mathcal{L}$, ordered by increasing real part, as,

$$
\operatorname{eig}(\mathcal{L})=\left\{\gamma_{0}, \gamma_{1}, \ldots, \gamma_{N-1}\right\}
$$

where $\gamma_{0}=0$. The following theorem is proven in [38].

Theorem 4: Given a plant, defined by (3) and (4), and $N$ parallel estimators/controllers, defined by (11) and (8),

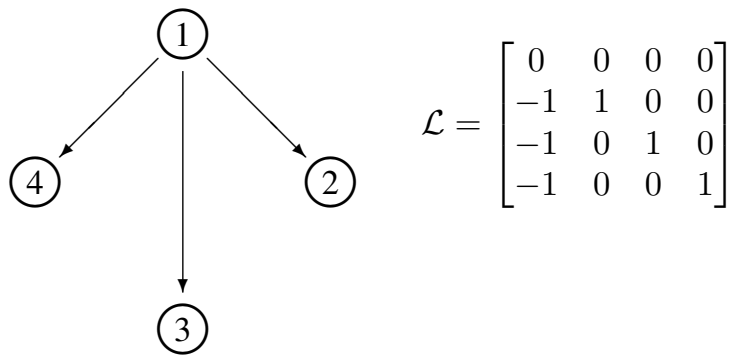

Fig. 4. Schematic illustration of the star topology with one transmitting and three receiving spacecraft. The Laplacian shown corresponds to the identical link case and has eigenvalues: $(0,1,1,1)$.

and with the communication between estimators defined by a single Laplacian, $\mathcal{L}$, the closed-loop system eigenvalues are:

$$
\begin{aligned}
\operatorname{eig}\left(A_{\text {clp }}\right) \cup \operatorname{eig}\left(A_{\text {est }}\right) \cup \operatorname{eig}\left(A_{\text {ctrl }}-\gamma_{1} F H\right) \cup \cdots \\
\cup \operatorname{eig}\left(A_{\text {ctrl }}-\gamma_{N-1} F H\right)
\end{aligned}
$$

We can see that in order to regain control of the disagreement dynamics, there must be $N-1$ non-zero eigenvalues of the Laplacian. This implies that the communication network must have at least $N-1$ receivers. $^{4}$

Another interesting case arises with a "star" topology illustrated in Figure 4. In this case the formation has a single transmitting spacecraft and $N-1$ receiving spacecraft. If each communication link has transmission and receiver matrices, $H_{l}, F_{l}, l=1, \ldots, N-1$, respectively, then the closed-loop eigenvalues are given by,

$$
\begin{aligned}
\operatorname{eig}\left(A_{\text {clp }}\right) \cup \operatorname{eig}\left(A_{\text {est }}\right) \cup \operatorname{eig}\left(A_{\text {ctrl }}-F_{1} H_{1}\right) \cup \ldots \\
\cup \operatorname{eig}\left(A_{\text {ctrl }}-F_{N-1} H_{N-1}\right) .
\end{aligned}
$$

In this topology, each communication link eliminates a single set of disagreement dynamics, making it a simple matter to completely specify the full closed-loop system dynamics.

These results allow us to draw some conclusions about the relative importance of transmitters and receivers in a formation's communication network. In order to remove the disagreement dynamics, $N-1$ receivers are required. However, only one transmitter is required and it does not matter which of the spacecraft transmits. This illustrates that the functioning of the receivers is more critical to the overall formation dynamics than the functioning of the transmitters. From an engineering point of view it is essential to invest in the reliability of the receivers within the network.

The result in Theorem 4 shows the the minimum amount of communication required to remove the disagreement dynamics grows linearly with the size of the formation. It is interesting, and not at all intuitive, that the star topology shows that the disagreement dynamics can be completely removed with only $N-1$ unidirectional communication links. Note that this result addresses only the stability and equilibrium dynamics of the system. The transient response of the formation depends also on the eigenstructure of $\mathcal{L}$ and may vary between formations having the same equilibrium dynamics.

${ }^{4}$ Strictly speaking it must have $N-1$ independent receivers. A closed cycle of $X$ links has only $X-1$ independent receivers. 
The results of this section give an alternative approach to that in Section III for implementing a distributed control system across the formation. This approach has the advantage that both measurement noise and channel noise can be explicitly accounted for within the design. Issues such as the loss of information on a single spacecraft (as illustrated in Figure 3) can be dealt with in a more straightforward and systematic manner. Further details can be found in [39].

\section{CONCLUSIONS}

The requirement for high precision in most spacecraft formation flying missions motivates control designs based on the complete formation state. In small formations measurement redundancy, plus communicated measurements to circumvent shadowing, may be sufficient to enable the implementation of the formation-wide control objective. In larger formations designs of this nature can be implemented by a single centralized estimator and controller, or by a distributed estimation architecture. Our focus is on the distributed estimation architecture for the following reasons: it does not have the performance limitations imposed by the round-trip communication latency; it improves the structural reliability of the formation by removing the central controller as a single point failure mode; and it gives each spacecraft sufficient information to implement higher level functions such as collision avoidance and formation manoeuvring.

Distributed estimation techniques must be used with care. Applying standard Kalman filter-based designs on each spacecraft leads to disagreement dynamics. The design of a complete distributed estimation scheme must also involve the modeling of the estimation errors on the other spacecraft in the formation. We have illustrated that this leads to a structured Kalman filter-based design problem.

An alternative approach is to design each estimator using standard Kalman filter-based methods, and then use communication between the spacecraft to reduce the effects of the disagreement dynamics. We have shown that at least $N-1$ spacecraft must receive information from one or more spacecraft in order to remove the effects of the disagreement dynamics. It is interesting to note that a star topology is capable of achieving this with one transmitter and $N-1$ receivers. This emphasizes the fact that receivers in the network play a more important role than transmitters and should have priority in reliability considerations.

The concept of disagreement dynamics can arise in other parallel estimation scenarios. One of interest is the use of redundant estimators in fault tolerant flight control systems. In such systems cross-linking is required to prevent the disagreement dynamics from driving the estimator differences beyond their allowed tolerances.

\section{ACKNOWLEDGMENTS}

The work described in this paper was carried out in part at the Jet Propulsion Laboratory, California Institute of Technology, under a contract with the National Aeronautics and Space Administration. The authors would like to thank Dr. David Bayard of JPL for helpful discussions on estimation.

\section{REFERENCES}

[1] G. Sholomitsky, O. Prilutsky, and V. Rodin, "Infra-red space interferometer," in 28th Int. Astro. Fed. Congress, 1977. Paper IAF-77-68.

[2] R. Stachnik, P. Melroy, D. Arnold, E. McCormack, and D. Gezari, "Multiple spacecraft Michelson stellar interferometer," in Instrumentation in Astronomy, V, Proc. SPIE 445, pp. 358-369, 1984.

[3] R. Stachnik and A. Labeyrie, "Astronomy from satellite clusters," Sky and Telescope, vol. 67, pp. 205-209, March 1984.

[4] A. B. DeCou, "Orbital station-keeping for multiple spacecraft interferometry," J. Astronaut. Sci, vol. 39, no. 3, pp. 283-297, 1991.

[5] G. B. Blackwood, S. Dubovitsky, R. P. Linfield, and P. W. Gorham, "Interferometer instrument design for New Millennium Deep Space 3," in Proc. SPIE 3350, p. 173, 1998.

[6] C. V. M. Fridlund, "Darwin-the infrared space interferometry mission," in ESA Bulletin, vol. 103, pp. 20-25, August 2000.

[7] K. Danzmann and LISA Study Team, LISA: Laser Interferometer Space Antenna for the detection and observation of gravitational waves. MaxPlanck Insitut für Quantenoptik, 2nd ed., July 1998.

[8] P. W. Gorham, W. M. Folkner, and G. B. Blackwood, "Enabling concepts for a dual spacecraft formation-flying optical interferometer for NASA's ST3 mission," in "Working on the Fringe", Proc. 1999 Dana Pt. Conf. on Optical Interferometry, PASP conf. series, vol. 194, pp. 395-400, Dec. 1999.

[9] P. R. Lawson, "The terrestrial planet finder," in Proc. IEEE Aerospace Conf., vol. 4, pp. 2005-2011, March 2001.

[10] K. Carpenter, C. Schrijver, R. Lyon, L. Mundy, R. Allen, J. Armstrong, W. Danchi, M. Karovska, J. Marzouk, L. Mazzuca, D. Mozurkewich, S. Neff, T. Pauls, J. Rajagopal, G. Solyar, and X. Zhang, "The Stellar Imager (SI) mission concept," in Proc. SPIE, vol. 4854, pp. 293-302, Aug. 2002.

[11] K. Gendreau, W. Cash, A. Shipley, and N. White, "The MAXIM pathfinder X-ray interferometry mission," in Proc. SPIE, vol. 4851, pp. 353-364, 2002.

[12] D. Quinn and D. Folta, "Tethered formation flying concept for the SPECS mission," Advances in Astronautical Sciences, vol. 104, pp. 183196, 2000.

[13] K. Mitsuda, H. Inoue, H. Kunieda, H. Yamakawa, H. Kuninaka, and T. Hashimoto, "Study of formation flying for the XEUS mission and the ISAS design of the detector spacecraft," in Proc. SPIE, vol. 4851, pp. 405-413, 2002.

[14] R. Bracewell, "Detecting nonsolar planets by spinning infrared interferometer," Nature, vol. 274, pp. 780-781, 24 Aug. 1978.

[15] F. P. Schloerb, "Imaging interferometry: lessons from the ground," SPIE Vol. 1947: Spaceborne Interferometry, pp. 249-260, 1993.

[16] K. Lau, S. Lichten, L. Young, and B. Haines, "An innovative deep space application of GPS technology for formation flying spacecraft," in AIAA Guidance, Navigation and Cont. Conf., July 1996. AIAA paper 96-3819.

[17] P. K. C. Wang and F. Y. Hadaegh, "Coordination and control of multiple microspacecraft moving in formation," J. Astronautical Sci., vol. 44 no. 3, pp. 315-355, 1996.

[18] F. Bauer, J. Bristow, D. Folta, K. Hartman, D. Quinn, and J. How, "Satellite formation flying using an innovative autonomous control system (AutoCon) environment," in AIAA Guidance, Navigation and Cont. Conf., pp. 657-666, 1997.

[19] F. Y. Hadaegh, W.-M. Lu, and P. K. C. Wang, "Adaptive control of formation flying spacecraft for interferometry," in Proc. 8th IFAC/IFORS/IMACS/IFIP Symp. on Large Scale Systems: Theory \& Appl. (P. Koussoulas, N.T.; Groumpos, ed.), vol. 1, pp. 117-122, Elsevier Sci., UK, July 1998.

[20] V. Kapila, A. G. Sparks, J. M. Buffington, and Q. Yan, "Spacecraft formation flying: Dynamics and control," in Proc. Amer. Control Conf., vol. 6, pp. 4137-4141, 1999.

[21] A. Robertson, G. Inalhan, and J. P. How, "Formation control strategies for a separated spacecraft interferometer," in Proc. Amer. Control Conf., vol. 6, pp. 4142-4146, 1999.

[22] D. Stansbery and J. R. Cloutier, "Nonlinear control of satellite formation flight," in AIAA Guidance, Navigation and Cont. Conf., August 2000. AIAA paper 2000-4436.

[23] P. K. C. Wang, J. Yee, and F. Y. Hadaegh, "Synchronized rotation of multiple autonomous spacecraft with rule-based controls: Experimental study," AIAA J. Guidance, Control and Dynamics, vol. 24, pp. 352-359, March-April 2001.

[24] M. Tillerson, L. Breger, and J. P. How, "Distributed coordination and control of formation flying spacecraft," in Proc. Amer. Control Conf., vol. 2, pp. 1740-1745, 2003. 
[25] X. Liu, A. Goldsmith, S. Mahal, and J. Hedrick, "Effects of communication delay on string stability of vehicle platoons," in Proc. IEEE Intell. Transport. Syst., pp. 625-630, 2000.

[26] M. R. Jovanović and B. Bamieh, "On the ill-posedness of certain vehicular platoon control problems," IEEE Trans. Auto. Control, 2005. (submitted).

[27] M. Mesbahi and F. Y. Hadaegh, "Formation flying of multiple spacecraft via graphs, matrix inequalities, and switching," AIAA J. Guidance, Control and Dynamics, vol. 24, pp. 369-377, March-April 2001.

[28] J. Fax and R. Murray, "Information flow and cooperative control of vehicle formations," in Proc. 15th IFAC World Congress, 2002.

[29] R. Olfati-Saber and R. Murray, "Graph rigidity and distributed formation stabilization of multiple-vehicle systems," in Proc. IEEE Control Decision Conf., vol. 3, pp. 2965-2971, 2002.

[30] P. O. Arambel, C. Rago, and R. K. Mehra, "Covariance intersection algorithm for distributed spacecraft state estimation," in Proc. Amer. Control Conf., vol. 6, pp. 4398-4403, 2001.

[31] F. Y. Hadaegh, B. H. Kang, and D. P. Scharf, "Rule-based estimation and control of formation flying spacecraft," in IEEE Conf. Fuzzy Syst., vol. 3, pp. 1331-1336, Dec. 2001.

[32] J. Lawton, A Behavior-Based Approach to Multiple Spacecraft Formation Flying. PhD thesis, Brigham Young Univ., 2000.

[33] J. Speyer, "Computation and transmission requirements for decentralized Linear-Quadratic-Gaussian control problems," IEEE Trans. Auto. Control, vol. 24, no. 2, pp. 266-269, 1979.

[34] J. R. Carpenter, "A preliminary investigation of decentralized control for satellite formations," in Proc. IEEE Aerospace Conf., vol. 7, pp. 63-74, 2000.

[35] P. Ferguson and J. How, "Decentralized estimation algorithms for formation flying spacecraft," in AIAA Guidance, Navigation and Cont. Conf., 2003. AIAA paper number: 2003-5442.

[36] D. M. Stipanović, G. Inalhan, R. Teo, and C. J. Tomlin, "Decentralized overlapping control of a formation of unmanned aerial vehicles," in Proc. IEEE Control Decision Conf., vol. 3, pp. 2829-2835, 2002.

[37] R. S. Smith and F. Y. Hadaegh, "Control of deep space formation flying spacecraft; relative sensing and switched information," AIAA $J$. Guidance, Control and Dynamics, vol. 28, no. 1, pp. 106-114, 2005.

[38] R. S. Smith and F. Y. Hadaegh, "Closed-loop dynamics of cooperative vehicle formations with parallel estimators and communication," IEEE Trans. Auto. Control, 2005. (submitted).

[39] R. S. Smith and F. Y. Hadaegh, "A distributed parallel estimation architecture for cooperative vehicle formation control," in Proc. Amer. Control Conf., 2006. (submitted).

[40] J. Fax and R. Murray, "Information flow and cooperative control of vehicle formations," IEEE Trans. Auto. Control, vol. 49, no. 9, pp. 14651476, 2004

[41] L. M. Pecora and T. L. Carroll, "Master stability functions for synchronized coupled systems," Phys. Review Lett., vol. 80, no. 10, pp. 21092112, 1998.

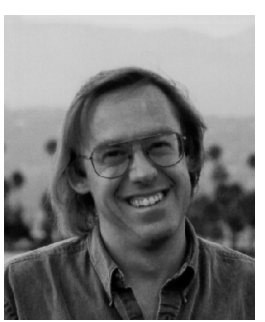

Prof. Roy S. Smith is with the Electrical \& Computer Engineering Department at the University of California, Santa Barbara. His research interests include: identification, modeling and robust control of feedback systems; distributed estimation and control; and design methodologies for uncertain constrained nonlinear systems. On the applications side, he has worked on a variety of experimental and simulation testbeds in the areas of: process control, automotive and marine engine systems, flexible space structures, aeromaneuvering Mars entry vehicles, formation flying of spacecraft, magnetically levitated bearings, and semiconductor fabrication facilities. Dr. Smith is a senior member of IEEE and AIAA, and a member of SIAM and NZAC.

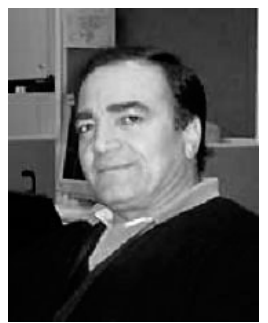

Dr. Fred Y. Hadaegh received both his BSEE with honors and his MSEE from the University of Texas at Austin and his Ph.D. in Electrical Engineering from the University of Southern California, Los Angeles. He joined the Jet Propulsion Laboratory, California Institute of Technology in 1984. He is currently a Senior Research Scientist and Technical Supervisor for the Guidance and Control Analysis Group. In addition, he is the Manager of the Distributed Spacecraft Technology Program at JPL. His research interests are in the areas of system identification, estimation theory and spacecraft control analysis and design. He has published extensively on mathematical modeling of uncertain systems, parameter identifiability, identification and control of large space structures, and autonomous control of formation flying space systems. He serves in various professional organizations and on technical committees. $\mathrm{He}$ is an Association Fellow of AIAA, Senior Member of IEEE, former Associate Editor of the IEEE Transactions on Control System Technology, and member of Eta Kappa Nu and Sigma Xi. He has received NASA's Exceptional Service Medal and NASA's Exceptional Achievement Medal. He has also received numerous NASA New Technology Awards. 